\title{
Parameters of left ventricular systolic and diastolic dyssynchrony on radionuclide imaging to improve cardiac resynchronization therapy in heart failure patients with dilated cardiomyopathy
}

\author{
Jayakumar Sreenivasan, MD, MSc, ${ }^{\text {a }}$ and Diwakar Jain, MD, FACC, MASNC ${ }^{\mathrm{a}, \mathrm{b}}$ \\ a Nuclear Cardiovascular Imaging Laboratory, Department of Cardiology, Westchester Medical \\ Center, Valhalla \\ b Department of Cardiovascular Medicine, New York Medical College, Westchester Medical \\ Center, Valhalla, NY
}

Received May 14, 2020; accepted May 14, 2020

doi: $10.1007 /$ s12350-020-02202-4

See related article, pp. 1023-1036

Cardiac resynchronization therapy (CRT) is a proven therapeutic intervention for selected patients with heart failure with reduced ejection fraction and electrical conduction delay. ${ }^{1}$ However, studies have shown that at least one-third of patients who undergo CRT based on currently recommended indications experience no significant improvement. ${ }^{2}$ Patient selection still remains a challenge despite multiple studies that have investigated predictors of favorable outcomes following CRT. ${ }^{3,4}$ Narrower baseline QRS duration, suboptimal left ventricular (LV) lead placement, larger myocardial scar burden, and ischemic cardiomyopathy are a few factors associated with poor response to CRT ${ }^{3-5}$ Multiple different parameters of LV mechanical dyssynchrony have been of recent interest to predict outcomes following CRT. ${ }^{6,7}$

Myocardial scar and non-viable myocardial segments can cause dyssynchronous LV contractility that does not adequately respond to CRT. ${ }^{6}$ Initial studies assessing LV dyssynchrony were mainly based on

\footnotetext{
Reprint requests: Diwakar Jain, MD, FACC, MASNC, Department of Cardiovascular Medicine, New York Medical College, Westchester Medical Center, 100 Woods Road, Valhalla, NY 10595; dj2700@gmail.com

J Nucl Cardiol 2021;28:1037-9.

$1071-3581 / \$ 34.00$

Copyright (c) 2020 American Society of Nuclear Cardiology.
}

conventional and tissue doppler echocardiography-based parameters like the timing of longitudinal myocardial velocity peaks. ${ }^{8}$ Several other echocardiographic parameters were found to be of interest in assessing the CRT response, however, those parameters had significant variability and had demonstrated only modest predictive power. ${ }^{8}$ A large study assessing novel echocardiographic findings like visually assessed apical rocking and septal flash as surrogates for myocardial dyssynchrony found that these parameters were associated with better survival following CRT. ${ }^{7}$ But a visual assessment by echocardiographic techniques is prone to intra- and interobserver variability, especially in patients with subtle patterns of dyssynchrony. ${ }^{7,9}$ In order to mitigate the limitations of echocardiography in dyssynchrony evaluation, phase analysis on gated singlephoton emission computed tomography (SPECT) myocardial perfusion imaging (MPI) has been studied as an alternative to echocardiographic assessment of LV systolic and diastolic dyssynchrony. ${ }^{10,11}$ Several studies have shown excellent correlation between the SPECTMPI and echocardiographic assessment of dyssynchrony. ${ }^{10,11}$ SPECT-MPI-based assessment of LV dyssynchrony relies on a demonstration of a wider spread in the timing of contraction and relaxation of various LV segments as compared to a narrower peak in normal hearts. The algorithms used for this assessment are highly automated with high reproducibility in contrast to echocardiography. ${ }^{12}$

It is intriguing that, it is not merely the systolic dyssynchrony, the diastolic dyssynchrony is also being increasingly suggested to be a contributor to poor 
response following CRT. ${ }^{13}$ A significant proportion of patients with systolic heart failure have been shown to have diastolic dyssynchrony in addition to the often sought out systolic LV dyssynchrony. ${ }^{13}$ Studies have shown varied findings in terms of changes in diastolic dyssynchrony pre and post CRT. ${ }^{13,14}$ However, the role of LV diastolic dyssynchrony in device responsiveness and functional outcomes is not adequately understood. Whether diastolic dyssynchrony is an independent variable or merely a reflection of systolic dyssynchrony is not entirely clear.

In this issue of the Journal, Wang et al report their findings on the predictive value of LV systolic and diastolic dyssynchrony in identifying treatment response among patients with dilated cardiomyopathy who underwent CRT. ${ }^{15}$ They evaluated 84 consecutive patients who underwent gated SPECT-MPI prior to CRT device implantation. Phase standard deviation and $95 \%$ width of phase histogram bandwidth were used to measure the global LV mechanical dyssynchrony. The primary outcome was CRT responsiveness defined as a $\geq 5 \%$ improvement in LV ejection fraction at 6-month follow-up. Study participants had a steep positive correlation between systolic and diastolic mechanical dyssynchrony. The study found that both systolic and diastolic mechanical dyssynchrony have incremental predictive value in addition to the conventional clinical predictors of CRT response including QRS duration, non-sustained ventricular tachycardia on telemetry, and optimal LV lead placement.

This study adds to the existing literature that in addition to the established clinical parameters, systolic as well as diastolic mechanical dyssynchrony assessment can help case selection for better prediction of optimal CRT outcomes. The study focused on diastolic dyssynchrony specifically, however, in the serial regression modeling, the diastolic dyssynchrony parameters in addition to systolic dyssynchrony abnormalities did not add significantly to the predictive power to the model in addition to the clinical predictors. The degree of myocardial scar burden which has known impact on CRT response and long-term outcomes was not significantly predictive of CRT responsiveness in this patient population, despite a larger proportion of subjects were reported to have scar burden. The authors have not adequately discussed this issue in their manuscript. Since the study population comprises only nonischemic cardiomyopathy patients, based on an absence of significant angiographic coronary artery disease, one does not expect a large scar burden in this population. However, various attenuation artifacts and a reduced septal radiotracer uptake in patients with LBBB can contribute to various degrees of apparent perfusion abnormalities. However, caution is required in interpreting these apparent abnormalities as indicative of scar.

Whether diastolic dyssynchrony is just a reflection of the systolic dyssynchrony in patients with severe cardiomyopathy rather than having an independent causal effect by itself on CRT outcomes remains unknown. Besides, it is important for future studies to assess the relationship of diastolic dyssynchrony and functional outcomes in terms of quality of life in patients receiving CRT as the current study did not assess the functional outcomes of patients at follow-up. In addition to dyssynchrony, the investigators showed that pacing at LV segments with both the late contraction and late relaxation as visualized on phase polar mapping was associated with better CRT response. This physiologically instinctive finding suggests the utility of phase analysis on gated SPECT-MPI for assessing optimal lead placement following CRT. Even though the study by Wang et al is limited by relatively small sample size, the findings are of important clinical implications in the selection of the most appropriate patients to expect the best outcomes following an invasive and expensive intervention like CRT. Study findings need to be validated over larger prospective cohorts with longer follow-up and clinical endpoints in addition to CRT responsiveness. Overall, this study definitely appeals to our curiosity to further explore currently available imaging techniques for better phenotypical characterization in addition to the clinical selection of patients to streamline the outcomes following CRT.

\section{Disclosures}

D. Jain is a speaker for Astellas and Pfyzer. Consultant for Astellas and GE. J. Sreenivasan has nothing to disclose.

\section{References}

1. Normand C, Linde C, Singh J, Dickstein K. Indications for cardiac resynchronization therapy. A comparison of the major international guidelines 2018;6:308-16.

2. Yu CM, Fung WH, Lin H, Zhang Q, Sanderson JE, Lau CP. Predictors of left ventricular reverse remodeling after cardiac resynchronization therapy for heart failure secondary to idiopathic dilated or ischemic cardiomyopathy. Am J Cardiol 2003;91:684-8.

3. Ypenburg C, van Bommel RJ, Delgado V, Mollema SA, Bleeker GB, Boersma E, et al. Optimal left ventricular lead position predicts reverse remodeling and survival after cardiac resynchronization therapy. J Am Coll Cardiol 2008;52:1402-9.

4. Sieniewicz BJ, Gould J, Porter B, Sidhu BS, Teall T, Webb J, et al. Understanding non-response to cardiac resynchronisation therapy: Common problems and potential solutions. Heart Fail Rev 2019;24:41-54.

5. Ypenburg C, Schalij MJ, Bleeker GB, Steendijk P, Boersma E, Dibbets-Schneider $\mathrm{P}$, et al. Impact of viability and scar tissue on 
response to cardiac resynchronization therapy in ischaemic heart failure patients. Eur Heart J 2006;28:33-41.

6. Beela AS, Ünlü S, Duchenne J, Ciarka A, Daraban AM, Kotrc M, et al. Assessment of mechanical dyssynchrony can improve the prognostic value of guideline-based patient selection for cardiac resynchronization therapy. Eur Heart $\mathrm{J}$ Cardiovasc Imaging 2018;20:66-74.

7. Stankovic I, Prinz C, Ciarka A, Daraban AM, Kotrc M, Aarones $\mathrm{M}$, et al. Relationship of visually assessed apical rocking and septal flash to response and long-term survival following cardiac resynchronization therapy (PREDICT-CRT). Eur Heart J Cardiovasc Imaging 2016;17:262-9.

8. Chung ES, Leon AR, Tavazzi L, Sun JP, Nihoyannopoulos P, Merlino J, et al. Results of the predictors of response to CRT (PROSPECT) trial. Circulation 2008;117:2608-16.

9. Jansen AH, van Dantzig J, Bracke F, Meijer A, Peels KH, van den Brink RB, et al. Qualitative observation of left ventricular multiphasic septal motion and septal-to-lateral apical shuffle predicts left ventricular reverse remodeling after cardiac resynchronization therapy. Am J Cardiol 2007;99:966-9.

10. Boogers MJ, Chen J, Veltman CE, van Bommel RJ, Mooyaart EA, Al Younis I, et al. Left ventricular diastolic dyssynchrony assessed with phase analysis of gated myocardial perfusion SPECT: A comparison with tissue Doppler imaging. Eur J Nucl Med Mol Imaging 2011;38:2031-9.

11. Hsu TH, Huang WS, Chen CC, Hung GU, Chen TC, Kao CH, et al. Left ventricular systolic and diastolic dyssynchrony assessed by phase analysis of gated SPECT myocardial perfusion imaging: A comparison with speckle tracking echocardiography. Ann Nucl Med 2013;27:764-71.

12. Fudim M, Dalgaard F, Fathallah M, Iskandrian AE, Borges-Neto S. Mechanical dyssynchrony: How do we measure it, what it means, and what we can do about it. J Nucl Cardiol 2019. https://d oi.org/10.1007/s12350-019-01758-0.

13. Schuster I, Habib G, Jego C, Thuny F, Avierinos JF, Derumeaux $\mathrm{G}$, et al. Diastolic asynchrony is more frequent than systolic asynchrony in dilated cardiomyopathy and is less improved by cardiac resynchronization therapy. J Am Coll Cardiol 2005;46:2250-7.

14. Alexanderson-Rosas E, Espinola-Zavaleta N, Garcia EV, Peix A, Massardo T, Pabon LM, et al. Diastolic dyssynchrony assessment by gated myocardial perfusion-SPECT in subjects who underwent cardiac resynchronization therapy. J Nucl Cardiol 2019. https://d oi.org/10.1007/s12350-019-01845-2.

15. Wang C, Shi J, Ge J, Tang H, He Z, Liu Y, et al. Left ventricular systolic and diastolic dyssynchrony to improve cardiac resynchronization therapy response in heart failure patients with dilated cardiomyopathy. J Nucl Cardiol 2020. https://doi.org/10.1007/s1 2350-020-02132-1.

Publisher's Note Springer Nature remains neutral with regard to jurisdictional claims in published maps and institutional affiliations. 